\title{
New Molecular Collisional Interaction Effect in Low-Energy Sputtering
}

\author{
Y. Yao, Z. Hargitai, M. Albert, R. G. Albridge, A. V. Barnes, J. M. Gilligan, B. Pratt Ferguson, \\ G. Lüpke, V. D. Gordon, and N. H. Tolk \\ Department of Physics and Astronomy, Vanderbilt University, Nashville, Tennessee 37235 \\ J. C. Tully \\ Department of Physics and Department of Chemistry, Yale University, New Haven, Connecticut 06520 \\ G. Betz and W. Husinsky \\ Institut für Allgemeine Physik, Technische Universität Wien, A-1040 Vienna, Austria
}

(Received 20 April 1998)

\begin{abstract}
An unexpected pronounced enhancement is observed in sputtering yields per atom for $\mathrm{N}_{2}^{+}$compared to $\mathrm{N}^{+}$from a polycrystalline gold target. This effect is seen when the kinetic energy per projectile atom is below $500 \mathrm{eV}$ and increases as projectile energy decreases to near-threshold energies. Enhancements for $\mathrm{O}_{2}^{+}$over $\mathrm{O}^{+}$begin at even lower kinetic energies below $100 \mathrm{eV}$ per atom. This new molecular interaction effect may be explained qualitatively by invoking a simple energy transfer model which involves the vibrational frequency of the molecule and the collisional interaction time. [S0031-9007(98)06668-X]
\end{abstract}

PACS numbers: 79.20.Rf, 34.50.Dy, 61.80.Lj, 81.15.Hi

We report on the first observations of a pronounced enhancement in sputtering yields for molecular ions as compared to atomic ions at low projectile energies near sputtering thresholds $(30-500 \mathrm{eV})$ [1]. At high energies $(\gtrsim 50 \mathrm{keV})$ sputtering yield enhancement with molecules has been observed and attributed to nonlinear effects in the collision cascade [2-4,5]. In the medium energy (linear cascade) regime (approximately $1-50 \mathrm{keV}$ ) it is known that the sputtering yield per atom of a molecular ion equals that of an atomic ion at the same impact velocity. In contrast, few data are available on sputtering yields at energies below $1 \mathrm{keV}$ (the low-energy regime). Furthermore, the literature that is available deals almost exclusively with atomic projectiles [6-13]. This work represents the first measurements of lowenergy sputtering yields by molecules near threshold, and shows an unexpected enhancement in sputtering yields for molecules as compared to atoms. This new effect can be attributed to the fact that the kinetic energy of a molecular projectile is most efficiently transferred when the time of collision is comparable to or larger than the vibrational period which is dependent on the detailed nature of the molecular potential. These experiments provide new and significant insight into fundamental projectile-surface interactions relevant to growth and etching.

The experiments were conducted in an ultrahigh vacuum chamber with a base pressure of $3 \times 10^{-9}$ Torr. Low-energy atomic and molecular ions were delivered by a unique accelerator that produces high fluxes of ions with energies tunable between $1 \mathrm{eV}$ and $2 \mathrm{keV}$. A schematic of the experiment is shown in the inset of Fig. 1. Ions were produced by a discharge source and focused by an Einzel lens. Mass separation was carried out by use of a Wien filter and neutral particles are eliminated by turning the beam by $1.3^{\circ}$ and passing it through a small aperture ( $4.8 \mathrm{~mm}$ diameter). The beam was then steered back onto the beam axis and refocused by a second Einzel lens. The beam was well defined spatially (FWHM $0.5 \mathrm{~mm}$ at $1 \mathrm{keV}$ ) and in energy (FWHM $1 \mathrm{eV}$ at $1 \mathrm{keV})$. The beam was scanned in a raster pattern over a mask with a $2 \mathrm{~mm}$ diameter aperture in order to have a homogeneous current density on the target. The current density was determined via an $80 \%$ transmission nickel mesh. The kinetic energy of the primary ions ranged from $10 \mathrm{eV}$ to $2 \mathrm{keV}$ with a current density $\geq 100 \mathrm{nA} / \mathrm{cm}^{2}$. Sputtering yields were acquired by monitoring the mass change of a goldcoated quartz-crystal microbalance (QCM). By using an

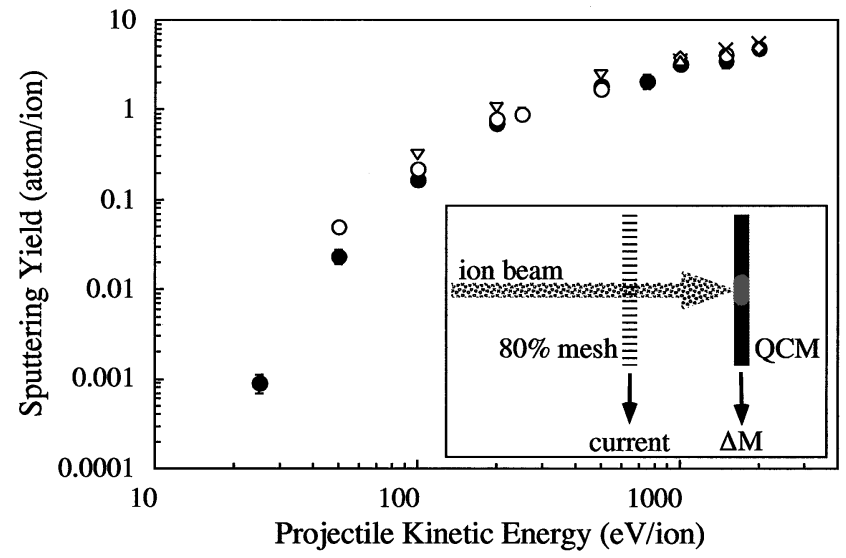

FIG. 1. Plot of the sputtering yields of $\mathrm{Ar}^{+}$on gold as a function of projectile kinetic energy from our experiment $(\bullet)$ and those from Wehner [13] ( $\triangle)$, Bates et al. [14] ( $\diamond)$, Laegreid and Wehner [15] $(\nabla)$, Robinson and Southern $[16](\times)$, and Colligon and Bramham [17] (o). The inset shows a schematic of the experimental configuration. 
aperture to mask the ion beam so that it only impinged on a $1 \mathrm{~mm}$ radius spot at the center of the QCM we were able to enhance the sensitivity of our QCM by a factor of 6 over the conventional method of illuminating the entire crystal, giving a mass sensitivity of about $2 \mathrm{ng} / \mathrm{cm}^{2}$ [11]. Figure 1 shows the sputtering yields of $\mathrm{Ar}^{+}$on gold as a function of projectile kinetic energy from our experiment and those from Wehner [13], Bates et al. [14], Laegreid and Wehner [15], Robinson and Southern [16], and Colligon and Bramham [17]. The uncertainties in our measurements are about $15 \%$.

The greatly enhanced sensitivity of the apparatus coupled with our low-energy, high-flux ion source allowed us to perform first-ever molecular-surface collision studies near sputtering thresholds. Figure 2(a) depicts the measured sputtering yields per atom of $\mathrm{N}_{2}^{+}$(open circles) and $\mathrm{N}^{+}$(closed circles) on a polycrystalline gold film as a function of projectile kinetic energy per atom at normal incidence. We normalized both the incident energy and the sputtering yield to the number of atoms in the projectile to study the difference between a single $\mathrm{N}_{2}^{+}$molecule and two unbound $\mathrm{N}^{+}$atoms. The sputtering yields per atom of $\mathrm{N}_{2}^{+}$and $\mathrm{N}^{+}$are essentially the same for energies above about $500 \mathrm{eV}$, as generally

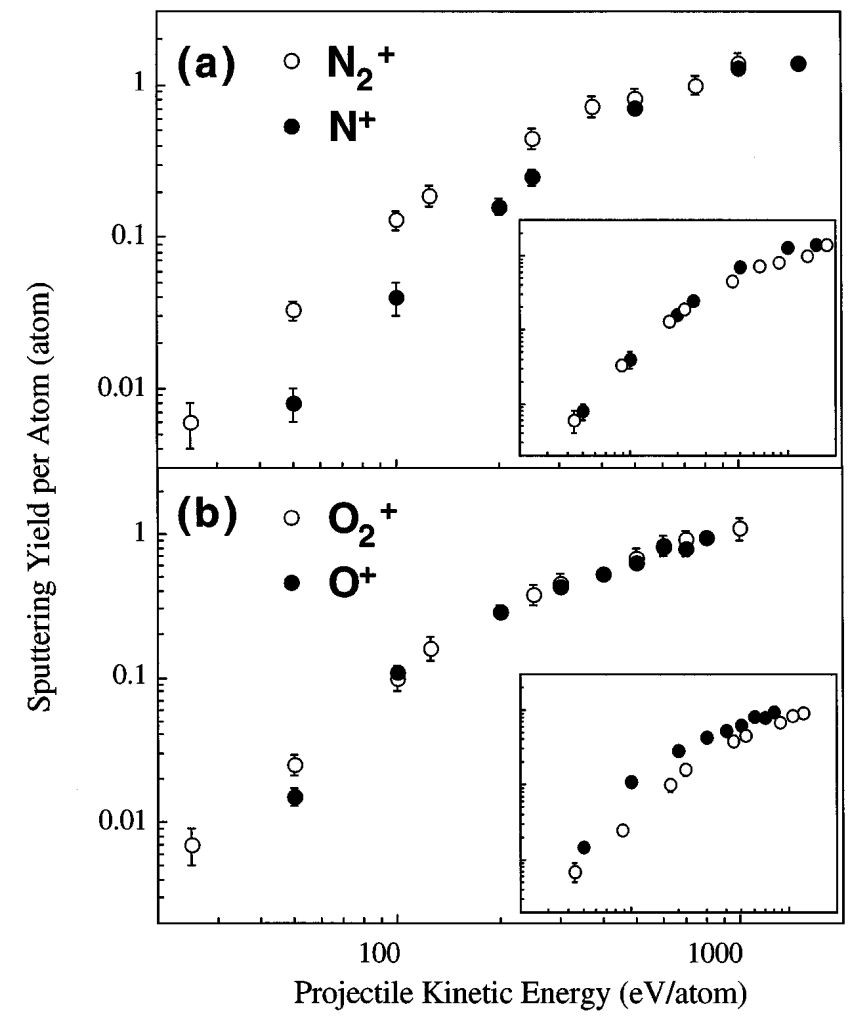

FIG. 2. Plot of the sputtering yield per atom of gold bombarded by (a) $\mathrm{N}_{2}^{+}$(o) and $\mathrm{N}^{+}$ions (•), and (b) $\mathrm{O}_{2}^{+}$(o) and $\mathrm{O}^{+}$ions $(\bullet)$ versus the projectile kinetic energy per atom. The insets show the same data plotted as a function of collisional energy transfer per atom, assuming the molecules are at the unified atom limit. expected. However, as the projectile kinetic energy decreases, the sputtering yields from $\mathrm{N}_{2}^{+}$diverge noticeably from the corresponding $\mathrm{N}^{+}$yields. This unexpected enhancement is noticeable at about $300 \mathrm{eV}$ and becomes more pronounced as the energy decreases further. The enhancement factor is approximately 4 at $50 \mathrm{eV}$.

To elucidate the nature of this new molecular effect, we performed similar studies with $\mathrm{O}_{2}^{+}$which has a different binding energy and stiffness (vibrational energy). Figure 2 (b) shows the sputtering yields per atom of $\mathrm{O}_{2}^{+}$(open circles) and $\mathrm{O}^{+}$(closed circles) which also shows the effect. Within experimental precision, the enhancement of the sputtering yield is observed at a projectile kinetic energy below $100 \mathrm{eV}$ per incoming atom. The enhancement factor is about 1.7 at $50 \mathrm{eV}$. An important feature of this experiment is that the onset of this new effect is observed at even lower energy than for nitrogen.

We may understand this effect qualitatively by invoking a simple model which takes into account (a) the amount of collisional energy transferred to a target atom by a diatomic molecule whose "effective mass" is dependent on vibrational frequency and collision time; (b) that the onset of this effect occurs when the half vibrational period equals approximately the collision time; and (c) the assumption that the low-energy ions are neutralized prior to their impact on the surface. In our analysis, we note that sputtering yield depends on the amount of energy transferred from an atom, $\Delta E_{\text {atom }}$, or a molecule, $\Delta E_{\text {molecule }}$, to a surface target atom. It is useful to treat two extreme cases. Let us consider two atoms of a diatomic molecule. In the limit of very large bond stiffness (high vibrational frequency), the molecular projectile may be considered as a single particle with a mass equal to the sum of the two atoms in the molecule. In the other limit, of a weak bond, the two atoms are not bonded. The maximum transferable energy per atom to a target atom through an elastic zero impact parameter collision is easily shown to be much larger in the first case than in the second. For elastic collisions between an atom (molecule) with mass $m(2 m)$ and kinetic energy $E_{0}\left(2 E_{0}\right)$ and a target at rest with mass $M$, the kinetic energy transferred to the target, $\Delta E$, scales as $\Delta E_{\text {atom }}=4 m M E_{0} /(m+M)^{2}$ $\left[\Delta E_{\text {molecule }}=4(2 m) M 2 E_{0} /(2 m+M)^{2}\right]$. The ratio of the energy transferred per atom for the two cases is

$$
\frac{\Delta E_{\text {molecule }}}{2 \Delta E_{\text {atom }}}=\frac{2(m+M)^{2}}{(2 m+M)^{2}} .
$$

Thus two ${ }^{14} \mathrm{~N}\left({ }^{16} \mathrm{O}\right)$ atoms with kinetic energy $E_{0}$ colliding with ${ }^{197} \mathrm{Au}$ atoms at rest will each transfer $0.248 E_{0}$ $\left(0.278 E_{0}\right)$, while an infinitely rigid $\mathrm{N}_{2}\left(\mathrm{O}_{2}\right)$ molecule, behaving as a single particle of mass 28 (32) with kinetic energy $2 E_{0}$ would transfer $0.436 E_{0}\left(0.481 E_{0}\right)$ per incident nitrogen (oxygen) atom. Thus the maximum energy transferred to a surface target atom is 1.76 (1.73) times greater for $\mathrm{N}_{2}\left(\mathrm{O}_{2}\right)$ molecules than for the atomic species. 
Normalized per atom in the projectile, the maximum energy that can be transferred to a surface atom will always lie between these two extreme cases. In a molecule-atom collision, a diatomic molecule $A B$ with mass $m_{A}+m_{B}$ may be considered as a single particle with some effective mass $m^{*}$, where $m_{A}, m_{B}<m^{*}<$ $m_{A}+m_{B}$. The precise value of the effective mass will depend, of course, upon the details of the collision, and particularly upon the projectile's kinetic energy. We make the assumption that $m^{*}$ will lie closer to the singleatom limit for fast collisions when the characteristic time of the collision interaction is much shorter than a half-period of the molecular vibration and that $m^{*}$ will lie closer to the rigid particle limit $\left(m_{A}+m_{B}\right)$ when the interaction time is much longer than a half-period of vibration. A similar argument comparing vibrational and collision times was used by Landau and Teller to explain energy transfer during gas phase molecular collisions [18].

The vibrational frequencies of $\mathrm{O}_{2}$ and $\mathrm{N}_{2}$ are 1554.7 and $2330.7 \mathrm{~cm}^{-1}$, respectively [19]. The corresponding vibrational half-periods are $1.1 \times 10^{-14} \mathrm{~s}$ for $\mathrm{O}_{2}$ and $7.2 \times$ $10^{-15} \mathrm{~s}$ for $\mathrm{N}_{2}$. To estimate the characteristic collision time we neglect the detailed interaction between the particles and simply assume a constant repulsive interaction force that turns on at some characteristic distance from the target. For simplicity, we consider a projectile with a small impact parameter, which decelerates to zero velocity in a distance of about $1 \AA$ and rebounds. In this case, the collision time is about $4 \AA / v$, where $v$ is the initial velocity of the projectile. Comparing the collision time to the vibrational half-period, we expect that the nonlinear enhancement should commence at kinetic energies of about $224 \mathrm{eV}$ per atom for $\mathrm{N}_{2}$ and $110 \mathrm{eV}$ per atom for $\mathrm{O}_{2}$. Figures 2(a) and 2(b) show enhancements starting at energies slightly above $300 \mathrm{eV}$ per atom for nitrogen and somewhere between 50 and $100 \mathrm{eV}$ per atom for oxygen, in reasonable agreement with the predictions of our simple model. At low impact energies, collision times will bring the interaction closer to the unified atom limit as revealed in the data.

The underlying assumption important for this model is that the sputtering yield is determined by the energy transferred to the surface atom. We test this model by comparing directly the sputtering yields of the atomic and molecular ions as a function of collisional energy transfer, as shown in the insets of Fig. 2. The energy transfer was computed for a zero impact parameter and assumes the molecules are at the unified atom limit. This has the effect of rescaling the projectile energy axis by a factor of 1.76 for $\mathrm{N}_{2}^{+}$and 1.73 for $\mathrm{O}_{2}^{+}$. We expect that this will yield a good agreement between the molecular and atomic data when the collision time is well below the vibrational halfperiod of the molecule (i.e., where the unified atom limit is valid). Indeed, the data show excellent agreement for projectile energies starting at the branching point where the atomic and molecular data separate (below $500 \mathrm{eV}$ for $\mathrm{N}_{2}^{+}$and below $100 \mathrm{eV}$ for $\mathrm{O}_{2}^{+}$).

To explore in more detail the novel features in our data, we have performed preliminary molecular dynamics (MD) calculations using a modified MD code which has been extensively tested and previously used for sputtering studies at higher bombarding energies [20,21]. As a first step we compared diatomic and atomic bombardment $(\mathrm{N}$ or $\mathrm{O})$ on a $\mathrm{Au}(111)$ surface, employing simple Morse potentials for the $\mathrm{Au}-\mathrm{Au}$ and the N-N (or O-O) interactions. These potentials were splined to the ZBL (Ziegler-Biersack-Littmark) potential at higher energies. A purely repulsive ZBL potential was used for the projectile atom-Au interaction. These preliminary calculations were in qualitative agreement with the observed experimental trends. In particular, at very low bombarding energies close to the sputtering threshold diatomic bombardment is found to always result in a higher sputtering yield than atomic bombardment. Further studies using more realistic potentials are under way to investigate in more detail the observed enhancement of sputtering yields at low projectile kinetic energies and to explore the possibility of a quantitatively predictive model.

In summary, we have presented the first observation of enhanced sputtering yields of molecular ions on a metal target at near-threshold energies. These effects are explained qualitatively by a simple model that compares the time scales of the molecular vibration and the collision interaction. Future work will involve strong synergy between MD simulations and experimental efforts. Silicon atoms provide the true unified atom limit for mass 28 , so we are studying it for comparison to $\mathrm{N}_{2}$ at low energies. Studies of NO and $\mathrm{CO}$ will be carried out to further characterize the roles of the vibrational frequency and the dissociation energy, particularly in $\mathrm{CO}$ where they vary in different directions compared to $\mathrm{N}_{2}$. Large cluster or molecule (e.g., $\mathrm{C}_{60}$ ) induced sputtering studies will also be carried out to study enhanced local energy transfer, which may be important to epitaxial growth mechanisms. Finally the dependence of sputtering yield on vibrational energy will be studied by using a free-electron laser to vibrationally excite both surface and projectile molecules during the sputtering process.

This work was supported by the Office of Naval Research under Grants No. N00014-94-1-1023 and No. N00014-94-10995 and by NASA under Grant No. NAG8-1128. The authors acknowledge helpful discussions with G. Margaritondo. G. Lüpke gratefully acknowledges financial support from the Alexander von Humboldt foundation.

[1] P. Sigmund, Phys. Rev. 184, 383 (1969).

[2] H. H. Andersen and H. L. Bay, in Sputtering by Particle Bombardment I, edited by R. Behrisch (Springer-Verlag, Berlin, 1981), p. 145. 
[3] P. Sigmund, in Sputtering by Particle Bombardment I, edited by R. Behrisch (Springer-Verlag, Berlin, 1981), p. 9.

[4] H.H. Andersen, in Fundamental Processes in Sputtering of Atoms and Molecules (SPUT 92), edited by P. Sigmund (Royal Danish Academy of Science and Letters, Copenhagen, 1993), p. 127.

[5] H. H. Andersen and H. L. Bay, Radiat. Eff. Defects Solids 19, 139 (1973).

[6] O.C. Yonts, Appl. Phys. 31, 1583 (1960).

[7] G. K. Wehner, Phys. Rev. 108, 35 (1957).

[8] R. V. Stuart and G. K. Wehner, J. Appl. Phys. 33, 2345 (1962).

[9] S. G. Askerov and L. A. Sena, Sov. Phys. Solid State 11, 1288 (1969).

[10] O. J. Orient et al., Phys. Rev. A 45, 2998 (1992).

[11] Y. Yao, Ph.D. dissertation, Vanderbilt University, 1997.

[12] Q. Yan, R. Albridge, A. V. Barnes, N. Seifert, and N. Tolk, Phys. Rev. B 55, 2854 (1997).
[13] G. K. Wehner, in Methods of Surface Analysis, edited by A. W. Czanderna (Elsevier, New York, 1975).

[14] J. Bates, D. M. Gruen, and R. Varma, Rev. Sci. Instrum. 47, 1506 (1976).

[15] N. Laegreid and G. K. Wehner, J. Appl. Phys. 32, 365 (1961).

[16] M. T. Robinson and A.L. Southern, J. Appl. Phys. 38, (1967).

[17] J. S. Colligon and R. W. Bramham, in Atomic Collisions in Solids, edited by D.W. Palmer, M.W. Thompson, and P.D. Townsend (North-Holland, Amsterdam, 1970), p. 258.

[18] L. D. Landau and E. Teller, Phys. Z. Sowjetunion 10, 34 (1936).

[19] G. Herzberg, in Spectra of Diatomic Molecules (Van Nostrand, New York, 1950), Vol. I, p. 62.

[20] G. Betz and W. Husinsky, Nucl. Instrum. Methods Phys. Res., Sect. B 102, 281 (1995).

[21] G. Betz and W. Husinsky, Nucl. Instrum. Methods Phys. Res., Sect. B 122, 311 (1997). 